\title{
A double blind trial of botulinum toxin " $A$ " in torticollis, with one year follow up
}

\author{
A P Moore, L D Blumhardt
}

\begin{abstract}
A double blind placebo controlled crossover trial was performed of botulinum toxin " $A$ " in 20 patients with spasmodic torticollis. There was a statistically significant benefit for those treated with toxin; 12 on toxin improved objectively, compared with four on placebo $(p<0.04)$. After a follow up period of one year, 16 still seemed to benefit from repeated toxin injections. The main side effect was dysphagia, which appeared to be dose related in individual patients.
\end{abstract}

Injection of botulinum toxin to cause temporary paralysis of selected muscles is becoming an accepted treatment for focal dystonias. In assessing benefit in spasmodic torticollis, open studies have reported uniformly good results, with objective response rates between $50 \%$ and $90 \%^{1-4}$ and even higher subjective response rates. Most double blind studies, however, have not produced such clear cut results. ${ }^{5-8}$ Tsui et al found objective improvement in $63 \%$ of subjects, with a placebo response in $33 \% .^{5}$ They interpreted this as being a statistically significant improvement with toxin, although this conclusion has been challenged. ${ }^{6}$ Gelb et al reported objective improvement with toxin in $60 \%$ of cases (substantial in only $35 \%$ ), but were unable to show objective superiority of toxin over placebo. ${ }^{6}$ Blackie and Lees found that toxin had a significantly larger effect than placebo with objective improvement in $74 \%$ of 19 patients on toxin, rated as moderate or marked in $37 \%$. Only one patient showed objective improvement on placebo. ${ }^{8}$ In a larger double blind study Greene et al found objective improvement in $61 \%$ of 28 patients given toxin, significantly greater than in the 27 placebo patients. The severity, duration and type of torticollis and the degree of jerkiness did not influence the response.

The subjective response rate has been between $66-80 \%$ in these controlled studies. In both open and blinded studies the abolition or improvement of neck pain has been consistently reported as a major benefit. Side effects have been relatively minor except for dysphagia, which is the main dose-limiting factor.

Each study has used a different treatment regime and this has hindered comparisons between them. Case selection and injection techniques, the use of EMG localisation, the dilution of the toxin and the total dose, have all varied. In addition, the American and
British toxins differ in potency and it has proved surprisingly difficult to establish their relative potencies. ${ }^{8}$

\section{Patients and methods}

A placebo controlled double blind crossover design was used. Twenty patients were included and their characteristics are shown in table 1. No patient had previously been treated with toxin or surgery and current drug regimes were not changed. We randomly allocated each patient on entry into two groups: group $A$, to receive toxin first at 0 and 2 weeks and placebo at 12 and 14 weeks, or group B, who were treated in the reverse order.

The hospital pharmacist prepared the toxin at a concentration of $5 \mathrm{ng} / \mathrm{ml}$. Visually identical vials of placebo were used. As a dose of $30 \mathrm{ng}$ of British toxin used by Stell et al had caused dysphagia in four out of 10 patients, ${ }^{3}$ we used a total dose of $25 \mathrm{ng}$ (1000 mouse units), giving it in two $12.5 \mathrm{ng}$ doses two weeks apart. This allowed the booster dose to be withheld if important dysphagia occurred.

At each injection session, the most active muscles were determined by assessing the type and direction of neck movement, muscle hypertrophy and visible or palpable contractions. The toxin was divided between the active muscles in approximate proportion to their estimated contribution to the dystonia. Some muscles received more than one injection.

Torticollis was rated objectively according to the scale used by Tsui et al. ${ }^{5}$

A) Amplitude of sustained movements: rotation, tilt and anteroposterior deviation of the head were scored individually as: $0=$ absent, $1=<15^{\circ}, 2=15-30^{\circ}, 3=>30^{\circ}$. The three scores were summed.

B) Duration of sustained movements: $1=$ intermittent, 2 = constant.

C) Shoulder elevation: $0=$ absent, $1=$ mild and intermittent, $2=$ mild and constant, $3=$ constant and severe.

D) Tremor: its severity: $1=$ mild: 2 = severe,

Table 1 Patient characteristics

\begin{tabular}{lll}
\hline & Group $A$ & Group $B$ \\
\hline Male:female & $5: 5$ & $4: 6$ \\
Mean age (years) & $48 \cdot 9(27-75)$ & $49 \cdot 6(35-61)$ \\
Mean age of onset (years) & $41.9(15-69)$ & $44.8(35-555)$ \\
Disease duration (years) & $6 \cdot 6(0 \cdot 25-30)$ & $6 \cdot 1(1-20)$ \\
Complex torticollis & 5 & 5 \\
Other dystonia & 6 & 3 \\
\hline
\end{tabular}


was multiplied by its duration: $1=$ occasional, $2=$ continuous.

Total score $=(\mathrm{A} \times \mathrm{B})+\mathrm{C}+\mathrm{D}$.

Torticollis was defined retrospectively as "complex", if the score for sustained movements A) was $>2$ in three planes or, $>2$ in two planes plus a tremor severity score $C$ ) of $>2$. An independent film crew recorded standardised videotapes at the start and at four weeks into each phase, as this was the expected time of maximum benefit. Patients were filmed seated at rest, whilst attempting to hold the head straight, and then turn it to each side, whilst writing, during natural and directed conversation and mental arithmetic, and whilst standing and walking, with both frontal and lateral views. The use of a geste antagonistique was persistently discouraged. The videos were later randomised before being scored by two blinded observers. The patients filled in a structured report of the subjective effects of treatment and clinical assessments were made at each visit.

Using the mean of the clinical scores and the two video scores at each visit, we defined objective change as a change in Tsui score of at least three points, or, of at least $30 \%$ if the baseline was less than eight. Results from the tremor subsection of the Tsui scale and the patients' reports of pain severity were compared between the active and placebo phases.

After one year in the follow up study patients were tested for the development of antibotulinum toxin antibodies using an ELISA test ${ }^{10}$ which is potentially more sensitive than the conventional biological assay. ${ }^{11}$ The test is suited to screening for the antibodies but is not a fully validated quantitative assay. ${ }^{12}$

\section{Results}

The two groups were evenly matched with respect to most relevant parameters (table 1), but more patients in group A had other dystonias. One patient in group B dropped out of the study after the two initial placebo injections, so that 19 completed the study. The correlation between observers using Tsui scores on the video recordings was 0.87 , but there was considerable scatter, sometimes as much as six Tsui points. Intra-observer variability was slightly worse $(r=0.64)$ when the video ratings and the clinic ratings were compared, but this may have been due to variation in the dystonia itself, as the video filming started between 10 and 30 minutes after the clinical rating.

Twelve patients responded objectively to toxin and four to placebo (Chi square $=5 \cdot 3$, $\mathrm{p}<0.05)$. Four patients in group B improved after both toxin and placebo. No patient deteriorated objectively after toxin, but two did so after placebo, one in group A probably because of declining toxin activity. Subjective improvement was still more significant, with 16 responding to toxin and three to placebo (Chi square $=15 \cdot 2, \mathrm{p}<0.01$ ).

The changes in mean Tsui video scores for the two groups in each period are shown in the figure. Group B showed a small placebo re-

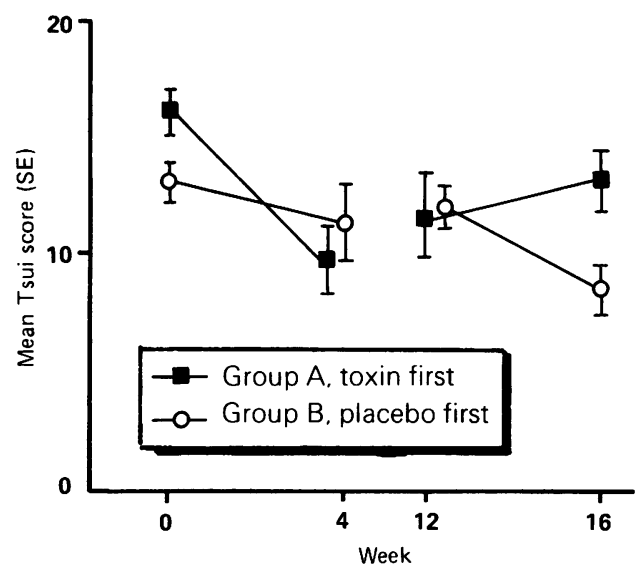

Figure Severity of torticollis at start and four weeks into each phase (high Tsui score = severe torticollis).

sponse in the first period, with greater improvement in the second period after active toxin. Group A showed a much larger improvement, returning towards baseline in the placebo phase. In retrospect, the washout period allowed between the two phases was probably too short. ANOVA was performed on the changes in Tsui video scores but there was no significant carryover $(\mathrm{F}=0.02, \mathrm{p}=0.89)$ or sequence $(F=2 \cdot 5, p=0 \cdot 14)$ effect. Most of the change in Tsui scores was attributable to the treatment $(F=6.9, p=0.02)$ and the mean difference between toxin and placebo was 4.2 Tsui points in favour of active toxin $(95 \%$ CI 0.79 to $7 \cdot 6$ ). The beneficial effect of toxin was usually apparent within five to 10 days of the first injection, but was occasionally not seen until a week after the second injection. There was useful benefit for eight to 10 weeks.

The difference between the mean (SD) improvement of tremor severity on toxin, 0.57 $(0.61)$, and on placebo, $0.05(0.54)$, was significant, $\mathrm{p}<0.01,95 \%$ CI 0.14 to 0.90 . Pain scores also fell more in the toxin than in the placebo phase but the difference failed to reach statistical significance.

The 19 patients who completed the double blind phase of the study were followed up by one of us (APM) in an open fashion for an average of one year and all except one decided initially to continue with the toxin. The remaining 18 showed little difference in benefit between those who responded objectively in the blinded part of the study and those who did not. Two of the 12 who responded objectively in the trial have stopped treatment because of inadequate subsequent benefit, but all six who did not respond have continued with the toxin. The mean interval between injections was three months. Table 2 shows the response to continuing treatment. Six patients had initially erratic benefit, partly because the doses were varied to minimise side effects. After one year of treatment none of the 11 patients who were tested by ELISA had developed antibodies to botulinum toxin. This included the patient who showed no continuing benefit and the two with declining benefit.

Dysphagia was the most important side effect (table 3), although it was never severe enough 
Table 2 Post trial benefit over one year

\begin{tabular}{lll}
\hline & \multicolumn{2}{l}{ Objective response } \\
in trial & \\
\hline & YES & NO \\
No post trial benefit & 1 & 0 \\
Declining despite increased dose & 2 & 0 \\
Occasionally good/usually minor & 4 & 2 \\
Steady with increasing dose & 2 & 0 \\
Usually good & 2 & 3 \\
Consistently good & 1 & 1 \\
\hline
\end{tabular}

Table 3 Adverse events during blinded study

\begin{tabular}{lll}
\hline & BOTOX & Placebo \\
\hline Dry/sore throat & 6 & 1 \\
Mild dysphagia & 6 & 0 \\
Moderate dysphagia & 1 & 0 \\
Local weakness & 5 & 0 \\
General weakness & 2 & 1 \\
Regional pain & 5 & 3 \\
Others & 9 & 6 \\
\hline
\end{tabular}

after the first injection to necessitate withholding the second dose. Dysphagia was defined as mild if there was clear difficulty in swallowing, and moderate if the diet had been modified. A dry or sore throat was common following toxin, but it was unclear whether this represented muscle weakness and dysphagia or autonomic side-effects of the toxin. Some of the 17 patients who continued with toxin were unpredictably liable to dysphagia for one to three weeks after a treatment session, but a recurrence could almost always be prevented by reducing the dose. Some patients noticed minor difficulties in raising their head off the bed, or felt the neck was weak for two to three weeks, but these symptoms were never disabling. Although the pain of torticollis was usually relieved, some patients had local discomfort from the injections and a few complained of a new pain around the neck or shoulder for a few weeks. We did not encounter episodes that suggested an immunologically mediated brachial plexopathy. ${ }^{13}$

\section{Discussion}

This study has demonstrated both subjective and objective benefit using botulinum toxin in spasmodic torticollis. The degree of improvement was substantial and all patients who improved chose to continue receiving the toxin despite the side effects. Initial failure to respond objectively did not preclude a good subsequent result. It is not clear why a number of previous blinded studies failed to show objective improvement, but possible reasons include the difficulty of rating torticollis and the use of different treatment regimes or injection techniques.

The most frequent side effect and dose limiting factor was dysphagia. This may be dose related in individual patients since in the open follow up study reducing the dose at the injection session following an episode of dysphagia almost always prevented another episode. Dysphagia may also be related to the precise placement of toxin and the extent of local diffusion. ${ }^{9}$ Occasional patients in previous trials have needed a nasogastric tube until their swallowing improved, ${ }^{3}$ but they had received larger doses of toxin than were used in this trial. There has also been one report to the Committee on Safety of Medicines of fatal inhalation pneumonia in a patient with severe chronic chest disease who received botulinum toxin for torticollis.

The best combination of toxin dilution, injection sites and timing is still unclear. Our ploy of splitting the dose into two worked just as well as previous regimes, but had no clear advantage in that seven of our 19 patients still developed dysphagia. We did not use EMG to identify the most active muscles and there is no evidence that it is necessary even in complex cases. It is generally assumed that more accurate injections are more effective, but it has not been proven that the agonist muscles have to be targetted precisely. After several courses of toxin, when it may be difficult to identify the active muscles, further injections still prove effective. Even if the effect of the toxin is purely peripheral, it may be that there is greater loss of function of those muscle fibres which are most abnormally active, as they more rapidly take up the toxin. ${ }^{14}$

Theoretically, it is possible that a general effect on central motor control mechanisms is as important as local paralysis. Most available evidence points to a central cause of dystonia. This includes studies of blink reflexes which have been shown to be abnormally excitable in both blepharospasm ${ }^{15}$ and isolated torticollis. ${ }^{16}$ In generalised dystonia, H-reflex studies have shown abnormal reciprocal inhibition of antagonist limb muscles. ${ }^{17}$ Clinical experience has shown that focal dystonia can be dispelled in some cases by weakening but not abolishing the equivalent voluntary movement. This is compatible with both a peripheral and central action of botulinum toxin.

Botulinum toxin could alter reflex abnormalities through a direct effect on central synapses. Centripetal axonal transport ${ }^{18}$ and transynaptic spread of toxin with entry into the spinal cord $^{19}$ have been demonstrated, but not a central synaptic effect. There is histological evidence of relative sparing of intrafusal muscle fibres in botulism, ${ }^{20}$ so that the toxin could also act by a differential toxicity in intra- and extrafusal muscle fibres resulting in alteration of the gain of the alpha-gamma link.

It is possible that decoupling of nervemuscle activity per se alters central processes. Section of a motor nerve trunk causes expression of hormones (pro-opiomelanocortin) in the analogous contralateral nerve terminals as well as the distal ipsilateral nerve terminals. The signal for this contralateral expression must pass via the spinal cord and is thought to be carried by slow axonal transport, because more proximal section of the motor nerve causes appropriately timed earlier contralateral hormone expression. ${ }^{21}$ Botulinum toxin could produce a similar effect.

Although many patients have now received multiple toxin injections the long term effects are unknown and there are no chronic toxicity 
studies in animals to guide us. Nevertheless, botulinum toxin is effective and appears to be relatively safe. Used with care it is an important advance in the management of focal dystonias.

1 Brin MF, Fahn S, Moskowitz C, et al. Localized injections of botulinum toxin for the treatment of focal dystonia and hemifacial spasm. In: Fahn S, Marsden CD, Calne DB, eds. Advances in neurology 50: Dystonia 2. New York: Raven Press, 1988:599-608.

2 Greene P, Shale H, Fahn S, Brin M, Friedman A. Treatmen of torticollis with injections of botulinum toxin. Neurology 1987;37 (suppl 1):123.

3 Stell R, Thompson PD, Marsden CD. Botulinum toxin in spasmodic torticollis. J Neurol Neurosurg Psychiatry spasmodic tortico
$1988 ; 51: 920-3$.

4 Jankovic J, Schwartz K, Donovan DT. Botulinum toxin treatment of cranial-cervical dystonia, spasmodic dysphonia, other focal dystonias and hemifacial spasm phonia, other focal dystonias and hemifacial

5 Tsui JKC, Eisen A, Stoessl AJ, Calne S, Calne DB. Doubleblind study of botulinum toxin in spasmodic torticollis. Lancet 1986;ii:245-7.

6 Gelb DJ, Lowenstein DH, Aminoff MJ. Controlled trial of botulinum toxin injections in the treatment of spasmodic torticollis. Neurology 1989;39:80-4.

7 Jankovic J. Blepharospasm and oromandibular-laryngealcervical dystonia: a controlled trial of botulinum A toxin therapy. In: Fahn S, Marsden CD, Calne DB, eds. Advances in neurology 50. Dystonia 2. New York: Raven Press, 1988:583-91.

8 Blackie JD, Lees AJ. Botulinum toxin treatment in spasmodic torticollis. J Neurol Neurosurg Psychiatry 1990; 53:640-3.

9 Greene P, Kang U, Fahn S, Brin M, Moskowitz C, Flaster
E. Double-blind, placebo-controlled trial of botulinum toxin for the treatment of torticollis. Neurology 1990;40: 1213-18.

10 Shone C, Appleton N, Wilton-Smith P, et al. Develop Biol Stand 1986;64:141-5.

11 British Pharmacopoeia. Biological assay of botulinum antitoxin. Appendix A152;1988

12 Hambleton P. Personal communication.

Glanzman RL, Gelb DJ, Drury I, Bromberg MB, Truong DD. Brachial plexopathy after botulinum toxin injections. Neurology 1990;40:1143.

14 Simpson LL. Botulinum neurotoxin and tetanus toxin. London: Academic Press, 1989:161.

15 Berardelli A, Rothwell JC, Day BL, Marsden CD. The pathophysiology of cranial dystonia. In: Fahn S, Marsden $\mathrm{CD}$, Calne DB, eds. Advances in neurology 50: Dystonia 2 . New York: Raven Press, 1988:525-35.

16 Tolosa E, Montserrat L, Bayes A. Blink reflex studies in patients with focal dystonia. In: Fahn $S$, Marsden CD, Calne DB, eds. Advances in neurology 50: Dystonia 2. New York: Raven Press, 1988:517-24.

17 Rothwell JC, Obeso JA, Day BL, Marsden CD. Pathophysiology of dystonias. In: Desmedt JE, ed. Motor control mechanisms in health and disease. New York: Raven control mechanisms in

18 Black JD, Dolly JO. Interaction of ${ }^{125} \mathrm{I}$-labelled botulinum neurotoxins with nerve terminals. 1: Ultrastructural autoradiographic localisation and quantitation of distinct membrane receptors for types $\mathrm{A}$ and $\mathrm{B}$ on motorneurones. $J$ Cell Biol 1986;103:521-34.

19 Wiegand H, Erdmann G, Wellhoner HH. ${ }^{125}$ I-labelled botulinum A neurotoxin: pharmacokinetics in cats after intramuscular injection. Naunyn-Schmiedebergs Arch Pharmacol 1976;292:161-5.

20 Sellin LC. The action of botulinum toxin at the neuromuscular junction. Medical Biology 1981;59:11-20.

21 Hughes S, Smith ME. Pro-opiomelanocortin-derived peptides in transected and contralateral motor nerves of peptides in transected and contralateral motor nerv 